\title{
High-pressure phase transition and equation of state of hydrous aluminous silica
}

\author{
GIACOMO CRINITI ${ }^{1}$, TAKAYUKI ISHII ${ }^{2}$, ALEXANDER \\ KURNOSOV $^{2}$, KONSTANTIN GLAZYRIN ${ }^{3}$ AND TIZIANA \\ BOFFA BALLARAN ${ }^{2}$ \\ ${ }^{1}$ Bayerisches Geoinstitut, Universität Bayreuth \\ ${ }^{2}$ University of Bayreuth \\ ${ }^{3}$ Deutsches Elektronen-Synchrotron \\ Presenting Author: giacomo.criniti@uni-bayreuth.de
}

Stishovite is the polymorph of $\mathrm{SiO}_{2}$ with tetragonal rutile structure that is stable in subducted basaltic lithologies at depths of the mantle transition zone and the lower mantle. It is known that stishovite undergoes a second-order ferroelastic transition to an orthorhombic $\mathrm{CaCl}_{2}$-type phase above $50 \mathrm{GPa}$ at room temperature, where extensive elastic shear softening is expected [1]. Previous studies showed that $\mathrm{Al}$ incorporation can considerably decrease the transition pressure [2], while it is still debated whether $\mathrm{H}$ has similar effects [3]. Here we report the equations of state, structural evolution and phase transformation of stishovite and $\mathrm{CaCl}_{2}$-structured samples having approx. 5 (A15) and $11 \mathrm{~mol} \%$ AlOOH (Al11). The All1 sample shows orthorhombic symmetry from ambient conditions up to $50 \mathrm{GPa}$, which is the maximum pressure investigated. A15, on the other hand, is tetragonal at ambient conditions and becomes orthorhombic upon compression at about $15 \mathrm{GPa}$. However, based on preliminary structural refinements and Raman spectroscopy measurements on Al5 at high pressure, the soft optic mode and octahedral tilting, which are related to elastic softening, remain the same as in the tetragonal phase until 20 GPa. Based on the observed different behaviors of spontaneous strains, octahedral tilting and soft optic mode in the $\mathrm{CaCl}_{2}$-type structure of $\mathrm{Al} 5$, we argue that $\mathrm{H}$ incorporation and $\mathrm{H}$-bond symmetrization may modify the character of the phase transition, and thus affect the elastic properties of stishovite and $\mathrm{CaCl}_{2}$-type in the lower mantle.

[1] Carpenter, Hemley \& Mao (2000), Journal of Geophysical Research, 105, 807-816.

[2] Lakshtanov, Sinogeikin, Litasov, Prakapenka, Hellwig, Wang, et al. (2007), Proceedings of the National Academy of Sciences of the United States of America, 104(34), 13588-13590.

[3] Umemoto, Kawamura, Hirose \& Wentzcovitch (2016), Physics of the Earth and Planetary Interiors, 255, 18-26. 